\title{
Photometric Observations of Three Double Stars by the Occultation Method - Comparison with Interferometric Results and with Preliminary Results of HIPPARCOS
}

\author{
M. FROESCHLÉ \& C. MEYER \\ OCA/CERGA, Avenue Copernic, F - 06130 Grasse, France
}

\begin{abstract}
We first briefly recall the geometry of the occultation of a double star by the Moon's edge. Then we give a short description of the principle of the formation of the diffraction pattern. We present the results for three double stars and compare them with those obtained by other methods.
\end{abstract}

\section{OCCULTATION GEOMETRY OF A DOUBLE STAR}

Figure 1 shows how the disappearance of a star behind the Moon's edge allows the observation of fringes for a ground-based observer. The same figure shows what occurs in the case of a double star: we observe the superposition of two fringe patterns, resulting in a typical signal when the separation of the components is sufficiently large ( $>3$ mas). If not, the interpretation of the signal does not always appear as obvious. The reductions of the observations are processed with a fitting method.
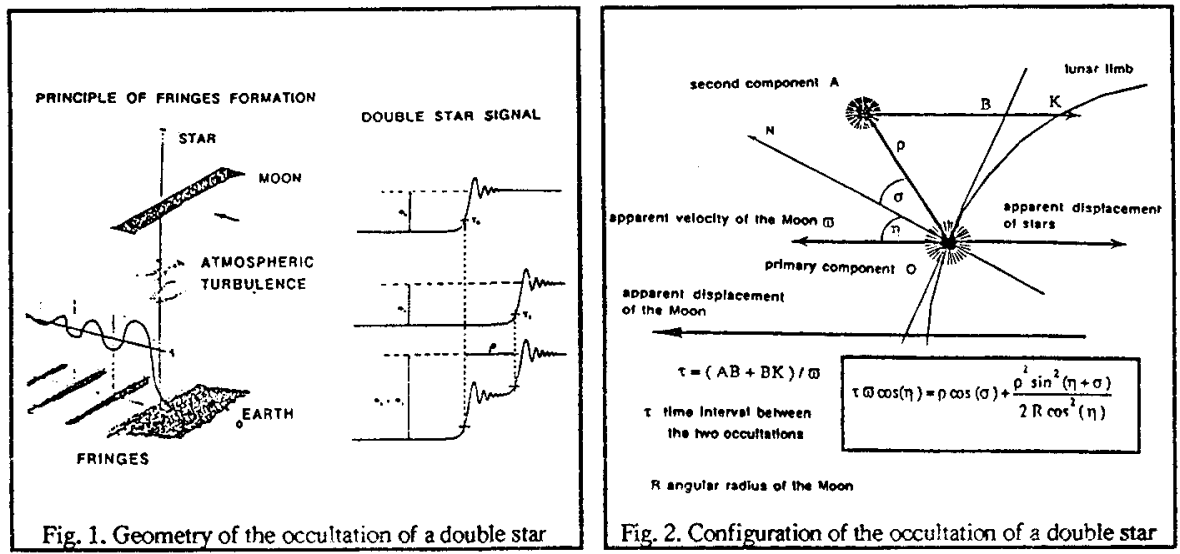

FIGURE 1. (left) Geometry of the occultation of a double star FIGURE 2. (right) Configuration of the occultation of a double star

In Figure 2 appear the different parameters that have to be taken into account in a reduction process. The observation itself consists of the estimation of the time $\tau$ that elapses between the occultations of the two components. The second term of the right-hand member of the equation is almost always negligible, particularly for the observations reported here. The result of a single reduction consists of a measure of the projected separation $\varrho \cos \sigma$ upon the 


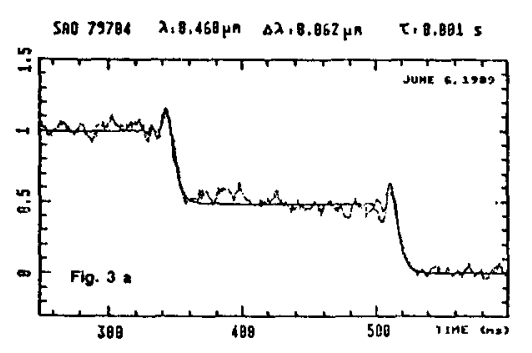

Dotreo lime , onsenunison (telac)

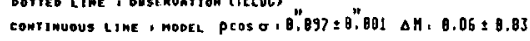

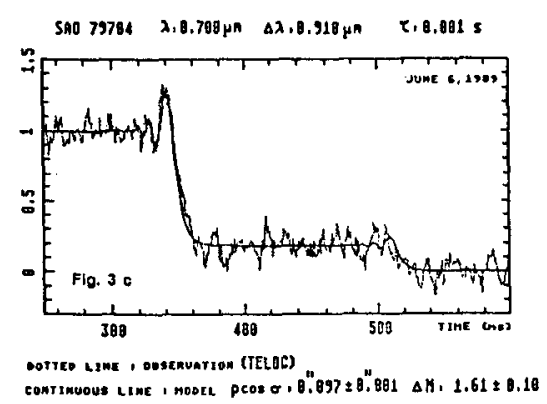

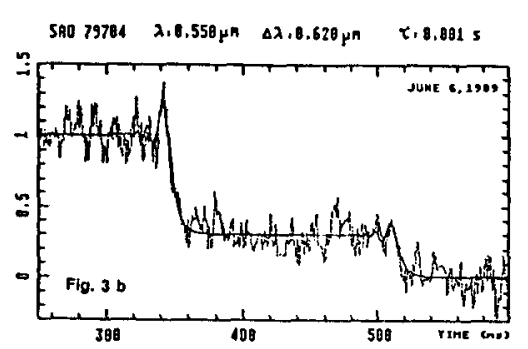

DOTJED LINE : Oesenvation (TELDC)

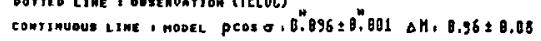

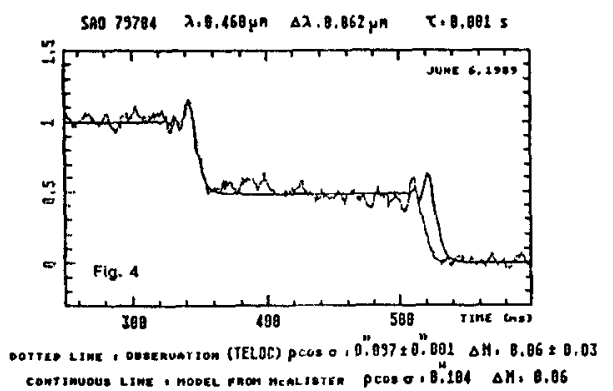

FIGURE 3 (top \& lower left) Occultation observations of SAO 79784 in 3 colors FIGURE 4 (lower right) Comparison with data from McAlister \& Hartkopf (1988)

perpendicular to the Moon's edge at the point of occultation, and also in an estimation of the difference in magnitudes between the two components.

\section{OBSERVATIONS AND RESULTS FOR 3 DOUBLE STARS}

The first one is the well-known triple star $82 \mathrm{Gem}=\mathrm{SA0} 79404$, often observed by interferometric methods. Figures $3 a, b, c$ show our observations of the system as a double star. The observations have been made in 3 colours, each giving a projected separation and a good estimate of the magnitude difference $\Delta M$. It is to be noted that the 3 values for the separation are very close and that there is no doubt that $\Delta M$ varies with the wavelength.

Our observation can be directly compared with interferometric determinations of separation which have been obtained by extrapolation of data for distance and angular position (McAlister \& Hartkopf 1988). It appears clearly in Figure 4 that the results differ significantly: $0^{\prime \prime} .007$ for $\varrho \cos \sigma$ (meaning $0^{\prime \prime} .017$ in true separation).

How we can explain such a difference? There are many possibilities to obtain a full agreement between the observations and the model: 


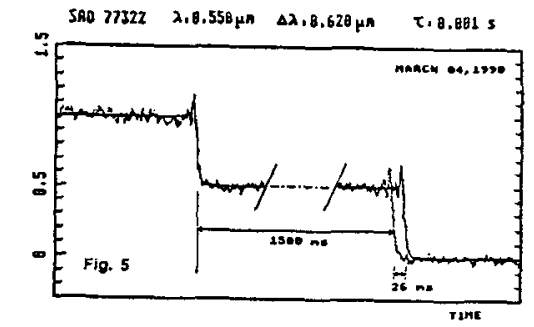

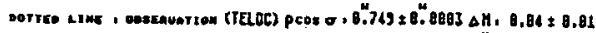

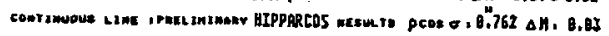

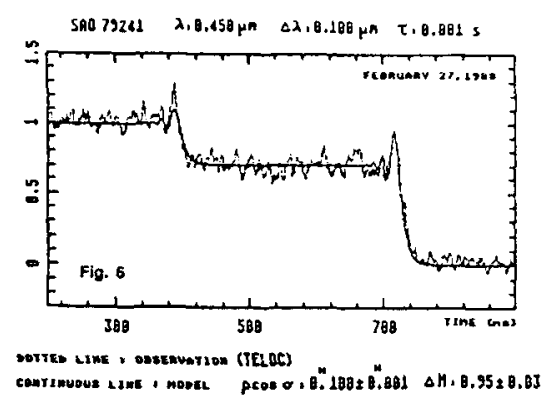

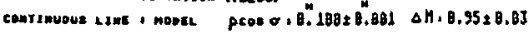

FIGURE 5. (left) Occultation observations of SAO 77322

FIGURE 6. (right) Occultation observations of SAO 79241

- a simple 12-m high cliff discontinuity on the lunar limb between the two disappearence points ( $500 \mathrm{~m}$ apart in this case) but far from each of them,

- a continuous slope of $1^{\circ} .4$ in the vicinity of the occultation,

- a error of $1: 4$ in position angle, which cannot be excluded,

- an error of 17 mas in interferometric distance measurements (very improbable hypothesis),

- or finally any plausible combination of the previous assumptions.

As a second example, Figure 5 shows the occultation light curve and an associated model derived from HIPPARCOS preliminary results by the FAST Consortium for the star SAO $77322=$ HIC 26396. We note the very good agreement on magnitude difference, but here again a significant disagreement on projected separations. The same explanations as above apply:

- discontinuity of $26 \mathrm{~m}$,

- continuous slope or error in position angle of 0.9 (the occultation points are separated by $1.7 \mathrm{~km}$ on the lunar limb),

- a very improbable error of 20 mas on the true separation.

The last example, Figure 6, is a good illustration of the case of a double star with an easily measurable separation and a $\Delta \mathrm{M}$ of only one magnitude, but not known as a double star at the time of construction of the HIPPARCOS Input Catalogue. Also observed elsewhere (Schmidtke et al. 1989) this star has evidently been detected as double by HIPPARCOS.

In conclusion, no method can be regarded as completely free of errors. However, lunar limb irregularities are probably the most important of the reasons that lead to a loss of precision in the resolution of double star systems by the occultation method. This is especially true in the case of a single determination.

\section{REFERENCES}

McAlister, H.A. \& Hartkopf, W.I., 1988 Second Catalog of interferometric measurements of binary stars, CHARA Contribution No. 2.

Schmidtke, P.C., Africano, J.L., \& Quigley, R., 1989, AJ, 97, 909 